\title{
Design of a SW Framework for an Autopilot with Automated Test Capabilities on an Experimental Mid-Sized UAS
}

\author{
E. De Lellis ${ }^{1}$, F. Corraro ${ }^{2}$, G. Di Capua ${ }^{3}$, L. Garbarino ${ }^{4}$, N. Genito ${ }^{5}$, R. Rocchio ${ }^{6}$ \\ CIRA - Italian Aerospace Research Centre, Capua (CE), 81043, Italy
}

In the framework of Clean Sky 2 LPA (Large Passenger Aircraft) Platform 1 program, CIRA is the leader of the WP1.3.5 aiming at developing an advanced Autopilot, and a dedicated testing framework for supporting the Dynamically Scaled Vehicle flight demonstrations. The presented Autopilot not only includes basic features like other commercial products, but also introduces new characteristics specifically designed for supporting scaled flight testing on unconventional A/C (aircraft) configurations, allowing implementation of advanced control laws architectures and strategies. To facilitate the flight testing, the framework integrates a dedicated SW function for automated tests, so avoiding, as much as possible, manual piloting and increasing test condition repeatability. Moreover, to facilitate future modifications, the framework includes a library of Simulink modules including basic guidance and control modules, and makes available to the final user the possibility to use a simple procedure for efficiently and reliably generating embedded real time code to be deployed on the on-board HW. Finally, the functional architecture has been designed to be compatible with the highest levels of autonomy with the aim to be easily integrated with additional autonomous functions, so to support future UAS (Unmanned Aerial Systems) operations. This goal has been pursued by developing a modular and scalar architecture and by adding the capability to execute complex automatic functions based on a simple flight instruction list defined by the remote pilot or the ground operator. To this end, the developed programming meta-language would allow performing mission tasks with better efficiency and effectiveness. For supporting and referencing these activities, a specific state of art study on autonomy classification has been conducted as well.

\section{Nomenclature}

$$
\begin{array}{ll}
\operatorname{deg} & =\text { degrees } \\
\min & =\text { minutes } \\
\mathrm{m} / \mathrm{s} & =\text { meter per second } \\
\mathrm{s} & =\text { seconds }
\end{array}
$$

\section{Introduction}

Essentially built upon the positive experiences of the Clean Sky SFWA (Smart Fixed Wing Aircraft) project, the Clean Sky 2 LPA (Large Passenger Aircraft) operational activities started in July 2014 in all three major work packages also called "Platforms". Platform 1 -Advanced Engine and Aircraft Configurations- will provide the development environment for the integration of the most fuel efficient propulsion concepts into the airframe targeting next generation short and medium range A/C (aircraft), In Platform 1, WP1.3 aims at validating Scaled Flight Testing as a viable means to de-risk disruptive $\mathrm{A} / \mathrm{C}$ technologies and $\mathrm{A} / \mathrm{C}$ configurations to high TRL (Technical Readiness Level). CIRA is the leader of the WP1.3.5 aiming at developing the A/C advanced Autopilot, and a dedicated testing framework for supporting the Dynamically Scaled Vehicle flight demonstrations.

\footnotetext{
${ }^{1}$ Systems Engineer, On Board Systems \& ATM, AIAA member.

${ }^{2}$ On Board Systems \& ATM, Deputy Head.

${ }^{3}$ Senior Researcher, On Board Systems \& ATM.

${ }^{4}$ Senior Researcher, On Board Systems \& ATM.

${ }^{5}$ Senior Researcher, On Board Systems \& ATM.

${ }^{6}$ Senior Researcher, On Board Systems \& ATM.
} 
Scaled flight testing has been a way of demonstrating new aeronautic technologies for many years. Because of the typical small dimensions of the A/C used for this kind of testing, the guidance and control equipment used for support scaled flight tests are the same of remotely piloted A/C or unmanned aerial systems. In these fields, only quite recently some commercial products have been introduced on the market that allow implementing guidance and control systems for both rotary and fixed wing $\mathrm{A} / \mathrm{C}$ configurations.

These products, typically named 'autopilots', offer a "full turkey" solution for remote piloting of an unmanned $\mathrm{A} / \mathrm{C}$ from a small ground station using a dedicated command and control radio link. In these systems, both manual and automatic flights are possible, whereas manual remote piloting is still typically performed using line-of-sight radio-control. Example of such products are: Micropilot [11] that seems to offer the most professional tools in this market segment, Ardupilot [12] based on the famous Arduino platform, and OpenPilot [13] which is an open source platform. Anyway, while the key advantages of such products rely on their compactness, affordability, configurability and easy-to-use HW/SW architecture, they have many limitations when used for unconventional A/C configurations and/or when special flight test procedures shall be executed. They are, in fact, only optimized for the most common $\mathrm{A} / \mathrm{C}$ configurations (e.g. quadcopters and conventional fixed wing A/C) in executing standard flight mission (i.e. surveying, loitering, etc.) with low-end navigation sensor quality. Thus, adopting one of these commercial available platforms requires very often a difficult product customization, with no guarantee of a successful result.

Instead of using standard available autopilots, in this project an HW/SW framework for and advanced A/P (Autopilot) an $\mathrm{A} / \mathrm{C}$ in the class of $150 \mathrm{Kg}$ Take-Off weight is proposed. Such framework not only includes the basic features already offered by the above mentioned commercial products, but introduces new characteristics specifically designed for supporting scaled flight testing on unconventional $\mathrm{A} / \mathrm{C}$ configurations, allowing implementation of advanced control laws architectures and strategies. Another key difference with other cited autopilots is the presence of a set of sensors for Navigation \& Control with higher performance and reliability.

The architecture of the Autopilot has been designed accordingly to the MBD (Model-Based Development) approach and by using the Mathworks environment with the objective of being able to integrate in the same SW both conventional control laws and new algorithm concepts at the same time.

To facilitate the flight testing, the framework integrates a dedicated SW function for automated tests, so avoiding, as much as possible, manual piloting and increasing test condition repeatability. Moreover, to facilitate future modifications, the framework includes a library of Simulink modules including basic guidance and control modules, and makes available to the final user the possibility to use a simple procedure for efficiently and reliably generating embedded real time code to be deployed on the on-board HW.

Last but not least characteristic of the framework is a functional and SW architecture that, even if the specific flight tests planned in this project do not need a high level of autonomy, is already designed to be compatible with the highest autonomy levels. This goal is pursued by developing a modular and scalar architecture and by adding the capability to execute complex automatic functions based on a simple flight instruction list defined by the remote pilot or the ground operator. To this end, the developed programming meta-language would allow performing mission tasks with better efficiency and effectiveness and, for the purpose of flight testing, with increased repeatability.

The implementation of different and adjustable degrees of autonomy is an essential step inside the roadmap for the development of UAS (Unmanned Aerial Systems). Many studies have been conducted to address specific issues that are key in supporting the proper implementation of the autonomy in robot and, more specifically, in UAS. For this reason, a state of art of recognized levels of autonomy for robots/UAS has been conducted to select an autonomy scale compatible and functional to the framework developed as described in Section III. The System Design is illustrated in Section IV, meanwhile preliminary test results of the real time simulations performed in CIRA laboratory are reported in Section V.

\section{State of Art on Autonomy Classification}

Sheridan [3] lays the foundation of a characterization of HRI (Human-Robots Interaction). He proposes a 10 level scale of degrees of automation known as Sheridan's Levels of Autonomous Decision-Making and Execution.

Boyd [2] tries to identify a model for the human process of decision-making pursuing the military objective to attempt to disrupt enemy decision-making processes. The model found is based on four categories, Observe, Orient, Decide, and Act interacting with each other in the so called Boyd's OODA loop (shown in Fig. 1). Two important characteristics of Boyd's system with respect to other four-tiered systems are the presence of feedback and implicit control. Feedback is the concept that decisions do not necessarily have to become actions. Decisions themselves can spark new analysis tasks or requests for new observations. Implicit control is the process that runs in the background. For an UAS the background process is the A/C GN\&C (Guidance, Navigation, and Control). This process has implicit control of the vehicle and will continue to carry out its programmed commands for the vehicle unless instructed 
otherwise by a human or autonomous manager. In a perfectly normal situation, an autonomous manager would never have to act, as the GN\&C system would perform as expected until there is an off-nominal current or predicted future observation. It is only in this context that autonomous manager would have to perform a complete OODA loop and send the act commands to the relevant parts of the vehicle.

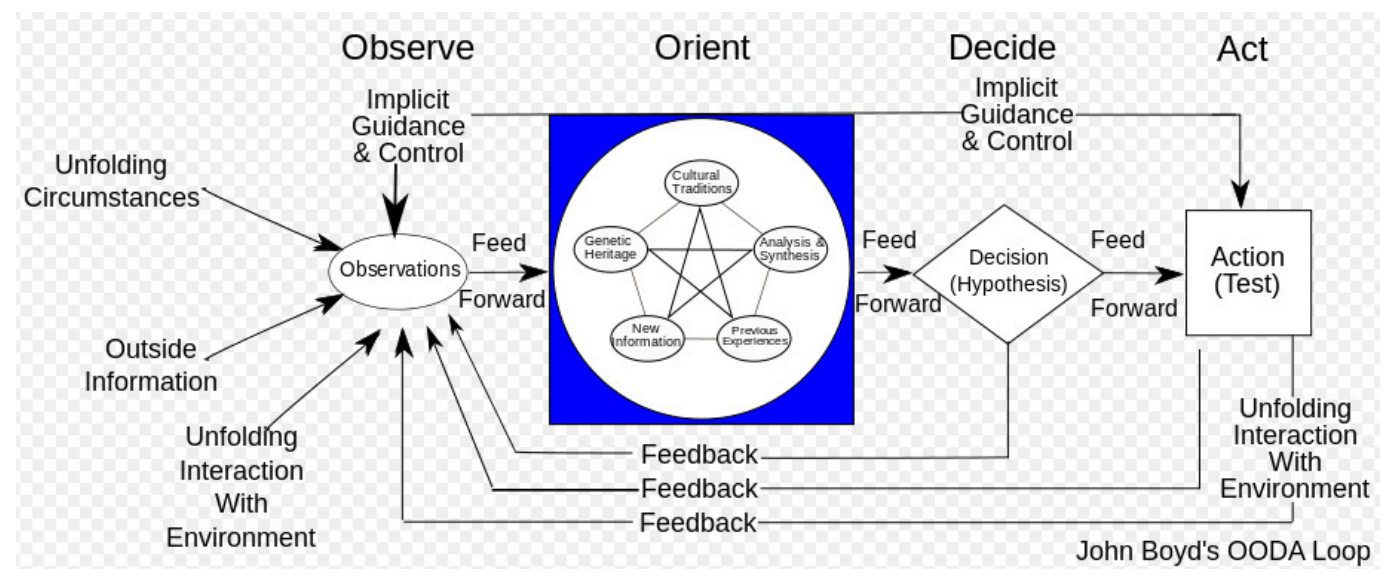

Fig. 1 - Boyd's OODA Loop [2].

Based on Boyd's loop, Clough [8] defines an interesting Autonomous Control Level (ACL) table that maps eleven levels of autonomy over the four descriptors represented by the steps of the OODA (Observe-Orient-Decide-Act) loop. This approach covers multi UAV operations that are dependent on the implementation of appropriate autonomy levels, but being the defined levels focused mostly on military/battleship situations they are not the most suitable for supporting the development of this project.

Proud et al. [4] highlight how often the literature assumes that the main goal of all autonomous systems is to strive for complete autonomy from human intervention, rather than identifying how autonomous each function within the system should have been. They develop a Level of Autonomy (LOA) scale (shown in Table 1) and an assessment tool to evaluate which level is the most appropriate for each function within a system. In order to capture effectively all the aspects of a decision-making system, the OODA terminology [2] for the level of autonomy functional types is very useful. Using OODA types enables the ability to break down known functions and to determine how autonomous to design each one. The defined LOA scales are bounded by levels 1 and 8, which correspond to complete human and complete computer responsibility, respectively (similar to Sheridan [3]), but each scale is tailored to fit the tasks encompassed by function type (Observe, Orient, Decide, or Act).

Generally, the levels of autonomy can be broken down into three sections. In Levels 1-2, the human is primary and the computer is secondary. In Levels 3-5, the computer operates with human interaction. In Levels 6-8, the computer operates independently of the human, and the human has decreasing access to information and decreasing override capability.

The NIST (National Institute of Standards and Technology) has set-up an ad-hoc group to address the autonomy issue. The group proposed a framework to manage ALFUS (Autonomy Levels for Unmanned Systems) [9],[10]. In their "detailed" model the autonomy level is determined by the following three factors:

- the complexity of the missions that an unmanned system is able to perform

- the degrees of difficulty of the environments within which the system can perform its missions

- the levels of operator interaction that are required to perform the missions.

Their final "summary" model proposes a negative linear correlation between LOA (and frequency of HI (Human Interaction), so that as the LOA increases, the HI frequency decreases. In his studies on the situational awareness within autonomous systems [6],[7], Hew also assumes that the levels of autonomy can be defined by the time interval between different human inputs/direction. Other reports, like [5], make the same assumption as well. 


\begin{tabular}{|c|c|c|c|c|}
\hline Level & Observe & Orient & Decide & Act \\
\hline 8 & $\begin{array}{l}\text { The computer gathers, filters, and } \\
\text { prioritizes data without } \\
\text { displaying any information to the } \\
\text { human. }\end{array}$ & $\begin{array}{l}\text { The computer predicts, interprets, } \\
\text { and integrates data into a result } \\
\text { which is not displayed to the human. }\end{array}$ & $\begin{array}{l}\text { The computer performs ranking } \\
\text { tasks. The computer performs final } \\
\text { ranking, but does not display results } \\
\text { to the human. }\end{array}$ & $\begin{array}{l}\text { Computer executes } \\
\text { automatically and does not } \\
\text { allow any human interaction. }\end{array}$ \\
\hline 7 & $\begin{array}{l}\text { The computer gathers, filters, and } \\
\text { prioritizes data without } \\
\text { displaying any information to the } \\
\text { human. Though, a "program } \\
\text { functioning" flag is displayed. }\end{array}$ & $\begin{array}{l}\text { The computer anlayzes, predicts, } \\
\text { interprets, and integrates data into a } \\
\text { result which is only displayed to the } \\
\text { human if result fits programmed } \\
\text { context (context dependant } \\
\text { summaries). }\end{array}$ & $\begin{array}{l}\text { The computer performs ranking } \\
\text { tasks. The computer performs final } \\
\text { ranking and displays a reduced set of } \\
\text { ranked options without displaying } \\
\text { "why" decisions were made to the } \\
\text { human. }\end{array}$ & $\begin{array}{l}\text { Computer executes } \\
\text { automatically and only } \\
\text { informs the human if required } \\
\text { by context. It allows for } \\
\text { override ability after } \\
\text { execution. Human is shadow } \\
\text { for contingencies. }\end{array}$ \\
\hline 6 & $\begin{array}{l}\text { The computer gathers, filters, and } \\
\text { prioritizes information displayed } \\
\text { to the human. }\end{array}$ & $\begin{array}{l}\text { The computer overlays predictions } \\
\text { with analysis and interprets the data. } \\
\text { The human is shown all results. }\end{array}$ & $\begin{array}{l}\text { The computer performs ranking tasks } \\
\text { and displays a reduced set of ranked } \\
\text { options while displaying "why" } \\
\text { decisions were made to the human. }\end{array}$ & $\begin{array}{l}\text { Computer executes } \\
\text { automatically, informs the } \\
\text { human, and allows for } \\
\text { override ability after } \\
\text { execution. Human is shadow } \\
\text { for contingencies. }\end{array}$ \\
\hline 5 & $\begin{array}{l}\text { The computer is responsible for } \\
\text { gathering the information for the } \\
\text { human, but it only displays non- } \\
\text { prioritized, filtered information. }\end{array}$ & $\begin{array}{l}\text { The computer overlays predictions } \\
\text { with analysis and interprets the data. } \\
\text { The human shadows the } \\
\text { interpretation for contingencies. }\end{array}$ & $\begin{array}{l}\text { The computer performs ranking } \\
\text { tasks. All results, including "why" } \\
\text { decisions were made, are displayed to } \\
\text { the human. }\end{array}$ & $\begin{array}{l}\text { Computer allows the human a } \\
\text { context-dependant restricted } \\
\text { time to veto before execution. } \\
\text { Human shadows for } \\
\text { contingencies. }\end{array}$ \\
\hline 4 & $\begin{array}{l}\text { The computer is responsible for } \\
\text { gathering the information for the } \\
\text { human and for displaying all } \\
\text { information, but it highlights the } \\
\text { non-prioritized, relevant } \\
\text { information for the user. }\end{array}$ & $\begin{array}{l}\text { The computer analyzes the data and } \\
\text { makes predictions, though the } \\
\text { human is responsible for } \\
\text { interpretation of the data. }\end{array}$ & $\begin{array}{l}\text { Both human and computer perform } \\
\text { ranking tasks, the results from the } \\
\text { computer are considered prime. }\end{array}$ & $\begin{array}{l}\text { Computer allows the human a } \\
\text { pre-programmed restricted } \\
\text { time to veto before execution. } \\
\text { Human shadows for } \\
\text { contingencies. }\end{array}$ \\
\hline 3 & $\begin{array}{l}\text { The computer is responsible for } \\
\text { gathering and displaying } \\
\text { unfiltered, unprioritized } \\
\text { information for the human. The } \\
\text { human still is the prime monitor } \\
\text { for all information. }\end{array}$ & $\begin{array}{l}\text { Computer is the prime source of } \\
\text { analysis and predictions, with } \\
\text { human shadow for contingencies. } \\
\text { The human is responsible for } \\
\text { interpretation of the data. }\end{array}$ & $\begin{array}{l}\text { Both human and computer perform } \\
\text { ranking tasks, the results from the } \\
\text { human are considered prime. }\end{array}$ & $\begin{array}{l}\text { Computer executes decision } \\
\text { after human approval. Human } \\
\text { shadows for contingencies. }\end{array}$ \\
\hline 2 & $\begin{array}{l}\text { Human is the prime source for } \\
\text { gathering and monitoring all data, } \\
\text { with computer shadow for } \\
\text { emergencies. }\end{array}$ & $\begin{array}{l}\text { Human is the prime source of } \\
\text { analysis and predictions, with } \\
\text { computer shadow for contingencies. } \\
\text { The human is responsible for } \\
\text { interpretation of the data. }\end{array}$ & $\begin{array}{l}\text { The human performs all ranking } \\
\text { tasks, but the computer can be used } \\
\text { as a tool for assistance. }\end{array}$ & $\begin{array}{l}\text { Human is the prime source of } \\
\text { execution, with computer } \\
\text { shadow for contingencies. }\end{array}$ \\
\hline 1 & $\begin{array}{l}\text { Human is the only source for } \\
\text { gathering and monitoring } \\
\text { (defined as filtering, prioritizing } \\
\text { and understanding) all data. }\end{array}$ & $\begin{array}{l}\text { Human is responsible for analyzing } \\
\text { all data, making predictions, and } \\
\text { interpretation of the data. }\end{array}$ & $\begin{array}{l}\text { The computer does not assist in or } \\
\text { perform ranking tasks. Human must } \\
\text { do it all. }\end{array}$ & $\begin{array}{l}\text { Human alone can execute } \\
\text { decision. }\end{array}$ \\
\hline
\end{tabular}

Table 1 - LOA (Level of Autonomy) Scale [4]

Beer et al. [1] highlight the difference between two schools of thought with which autonomy and HRI have been conceptualized:

- higher robot autonomy requires less frequent HRI

- $\quad$ higher robot autonomy requires higher levels of HRI.

The first viewpoint, that higher autonomy requires less HRI, has been supported by [5],[6],[7],[9], and [10]. The idea that higher autonomy reduces the frequency of interaction is a stark contrast to the other school of thought in which HRI researchers have proposed that more robot autonomy enables more sophisticated interaction. In this case, autonomy is proposed as a benchmark for developing social interaction in socially assistive robotics.

Based on all the previous considerations, the most appropriate way to classify the functions of the Autopilot described in this paper and the levels of autonomy achieved and achievable by the framework described is the one shown in Table 1 and introduced in [4]. 


\section{System Design}

The A/P system's main objectives are:

- to facilitate the program's flight test campaign execution

- to allow fast modification and/or re-tuning of the Flight Control Laws

- to ensure accurate and repeatable flight test conditions

- to develop a SW architecture compatible with adding or modifying functions for achieving, if required, the highest autonomy levels.

To pursue those objectives, some system design choices have been made and will be described in the following paragraphs of this section.

The A/P system is composed of two segments: the On-board A/P and the GRPS (Ground Remote Pilot Station).

\section{A. Rapid Prototyping Process by Using Recent Matlab/Simulink Tools}

A SW rapid prototyping process has been adopted based on automatic code generation from graphical low level specifications (i.e. Matlab/Simulink models) and on a custom library of standard control and guidance building blocks. A further characteristic of such environment consists in making available an easy tuning of parameters with no SW change needed between different flight tests or even between different phases of the same flight test.

The SW developed following a MBD approach will be shared among all the partners of the project. For this reason, having a collaborative design framework is essential for a successful project outcome. Such a framework provides team members with tools that enable efficiency, componentization, reuse, and knowledge transfer across the consortium. To develop a collaborative framework, the Simulink Project toolset has been exploited that makes easy to ensure a common application of standards such as company tools, libraries, and standard startup and shutdown scripts. Moreover, this toolset enables an efficient integration with source control and configuration management tools.

The chosen Simulink Project folder structure, depicted in Fig. 2, allows a rational storing of all the files by exploiting a logical organization based on the functional dependencies. The folder structure is composed by:

- $\quad$ simulinkProject folder for Matlab files related to the Simulink Project toolset

- common folder for variables, initialization and enumerative declaration common to all the functions

- function folder containing the Simulink models, nested as in the SW architecture. Each of those .slx files is called by the top-level model as a reference model. Also, this folder contains initialization scripts and local variables for each specific function

- mainModel folder containing the top level Simulink model from witch is possible to auto-generate the code and navigate in all the function's models

- $\quad$ source folder containing all the generated code files from the Simulink models

- test folder containing the test harnesses to be used for testing single functions or functionalities.

This structure is exploited to manage and take under configuration control the SW files, including the Simulink models, for the on-board SW application, the ground remote pilot station SW application, and the A/C simulator application.

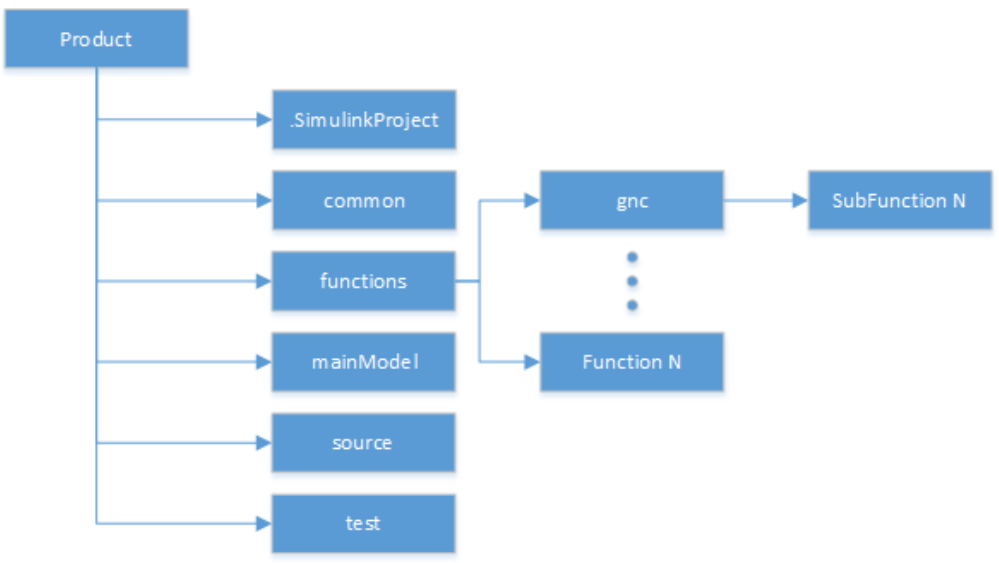

Fig. 2 - Development and Collaborative Framework -File Structure-. 


\section{B. Modular and Scalable Architecture}

The base architecture adopted for this A/P is a heritage of years of CIRA experience in developing autonomous systems. Even if the architecture has not been developed by explicitly taking as reference Boyd's OODA loop, the different functions developed for CIRA autonomous systems can be easily classified within the three OODA categories. An example of such classification is shown in Fig. 3.

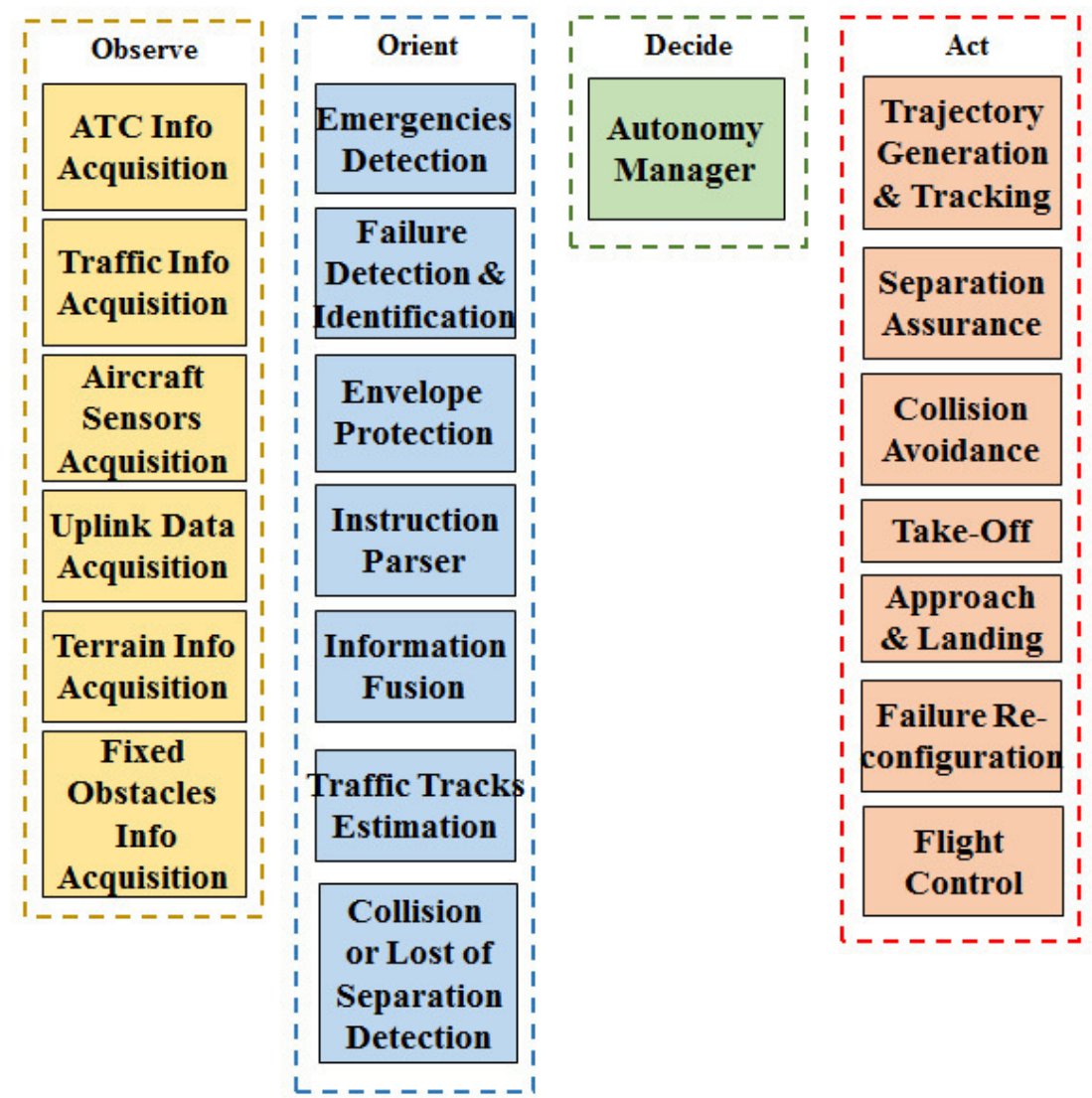

Fig. 3 - Autonomous functions classification into OODA paradigm.

The specific on-board A/P functional architecture (shown in Fig. 4) is designed to be modular and scalable by implementing the following features:

- $\quad$ an on-line configurable control law section composed by 'black-box' control modules

- a library of pre-defined 'flight modes' that will be in charge of activating the needed control laws as the specific test or flight phase requires

- the capability to expand the flight modes module by using a set of User Flight Modes directly added by the user by means of an on-board SDK (SW Development Kit)

- a test automation logic that executes a set of high level instructions for activating a sequence of flight modes as required by the test procedure

- an Inertial Navigation function implementing a Kalman Filter based on Inertial Sensors and GPS

- a Ground Remote Piloting function whose sub-functional decomposition is depicted in Fig. 5

- a set of control laws that includes standard control laws (i.e. laws based on standard control approaches like Proportional-Integrative-Derivative), advanced control laws (i.e. non-standard control approaches aiming at increasing control accuracy, dynamic performance and robustness to uncertainties)

- the capability to expand the Control Laws module by using a set of User Control Laws directly added by the user by means an on-board SDK. 
Taking [4] as reference, the autonomy level reached in this project would be high (6-8) for the observe type and medium (3-5) for the other types (i.e. orient, decide, and act). On the other hand, the proposed SW architecture can be used to reach the maximum level in every function type. In this project the instruction parser and the mission logic execute flight plan, automated test maneuvers, and manage emergencies in case the remote pilot cannot. Although, they could fully substitute the remote pilot in every task, potentially managing instructions from the ATC, avoiding obstacles and threats, completing mission high-level objectives and interacting with human operators at a high level by using a meta-language. This last kind of HRI is considered in CIRA vision and accordingly with all the considerations on HRI highlighted in [1], typical of a high autonomy level system.

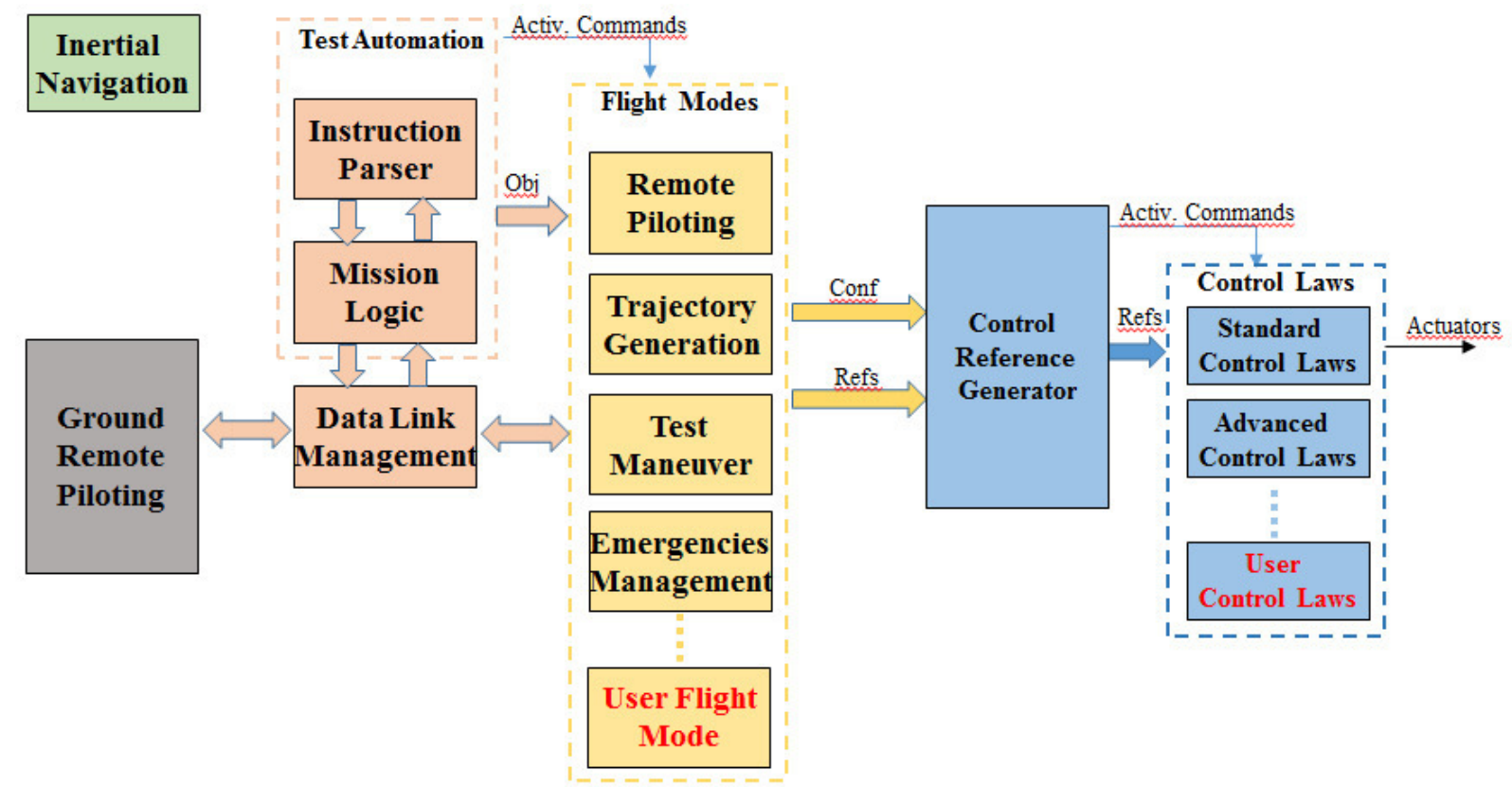

Fig. 4 - On-board A/P Functional Architecture.

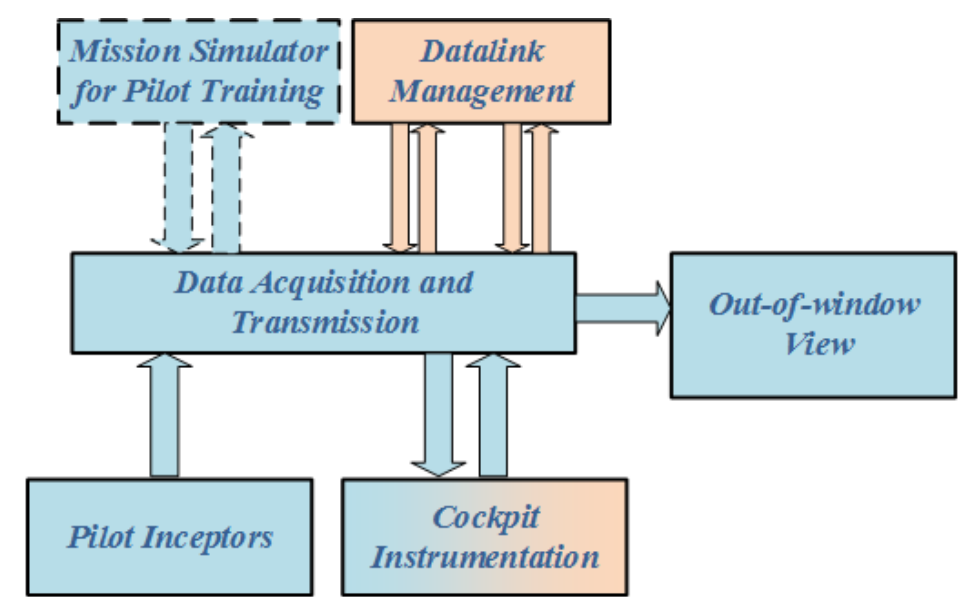

Fig. 5 - GRPS Functional Architecture.

\section{Flight Test Automation Language}

A dedicated SW function for automating test execution, based on a specific pre-compiled test instructions list, has been implemented. This allows performing several flight tests in the same conditions (thus easing results comparison), while still having manual remote pilot capabilities for both safety or dedicated maneuvers. Moreover, the current development would be the first step of the meta-language paradigm mentioned in the previous paragraphs. 
Every instruction aims to achieve a specific target, it could be a WP (Way Point) to be captured or a certain maneuver or test to be executed. Every instruction contains the parameters indicating which flight mode or control module combination, among the ones available, should be used in performing that instruction. This capability maximizes the benefits deriving from a scalable and modular architecture.

The test automation instructions are coded in a XML file containing all the instructions' lists selectable during the flight test. At the beginning of the mission, this file needs to be loaded on the on-board A/P by means of a specific interface available in the Cockpit Instrumentation HMI. During the flight test, the Remote Pilot will be able to modify the current lists or to generate a new one. Those changes will not modify the initial XML file that can, on the other hand, always be updated and re-downloaded overwriting the possible changes made during the flight.

All the currently available instructions has a target WP and can have a specified maneuver to be performed meanwhile the vehicle is tracking the leg towards a certain WP. An example of instruction list is shown in the Table 2 and Fig. 1.

\begin{tabular}{|l|l|l|}
\hline \multicolumn{1}{|c|}{ Target } & \multicolumn{1}{c|}{ Maneuver } & \multicolumn{1}{c|}{ Variable } \\
\hline WP1 & none & none \\
\hline WP2 & singlet & IAS \\
\hline WP3 & none & none \\
\hline WP4 & singlet & altitude \\
\hline
\end{tabular}

Table 2 - Example of List of Instructions for the Test Automation

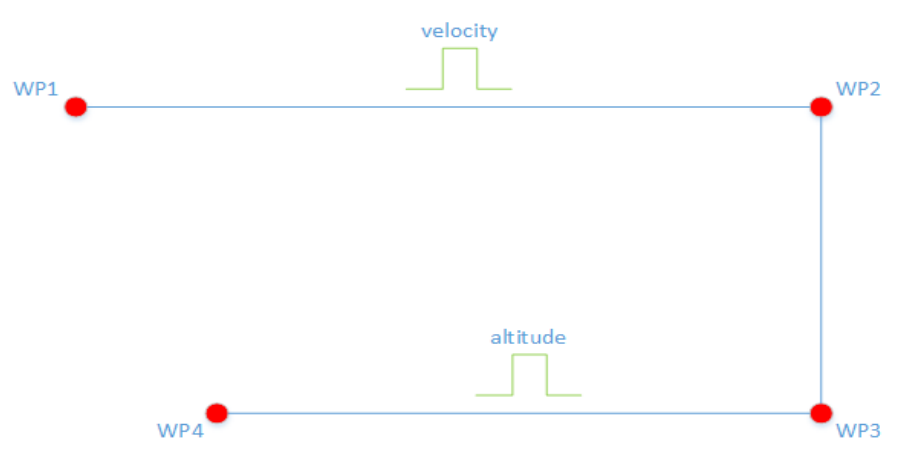

Fig. 6 - Example of Flight Plan for the Test Automation.

\section{Remote Pilot Training Integrated in the Ground Control Station}

The Ground Remote Pilot Station (GRPS) functional architecture has been developed to allow an efficient interaction between the Remote Pilot and the features made available by the A/P, a proper communication between the on-board and on-ground segments, and the management of the streaming video coming from the on-board forward camera and dedicated to the cockpit OTW (out-of-window) visualization. The Virtual Cockpit and OTW displays are shown in Fig. 7, meanwhile the GRPS layout is depicted in Fig. 8.

Furthermore, the same station can be used, in combination with a real-time simulator model implemented in the GRPS OGC (On Ground Computer), for verifying the mission execution and remote pilot training.

\section{System Test}

Some preliminary RT (Real Time) HIL (Hardware-In-the-Loop) simulations have been performed for verifying the system developed. The final laboratory tests will be done in Holland, once the system is delivered to NLR facility for integrating it on the flight demonstrator. Two campaign of flight tests are expected to be performed over the next two years on two different versions of the flight demonstrator both in Holland and in Italy.

Specifically, the capability to follow a list of automatic test instructions has been verified. The simplified list of flight test instructions executed during this test is:

- Go to WP1 and meanwhile perform a track angle singlet

- Go to WP2

- Go to WP3 and meanwhile perform a pitch angle singlet. 


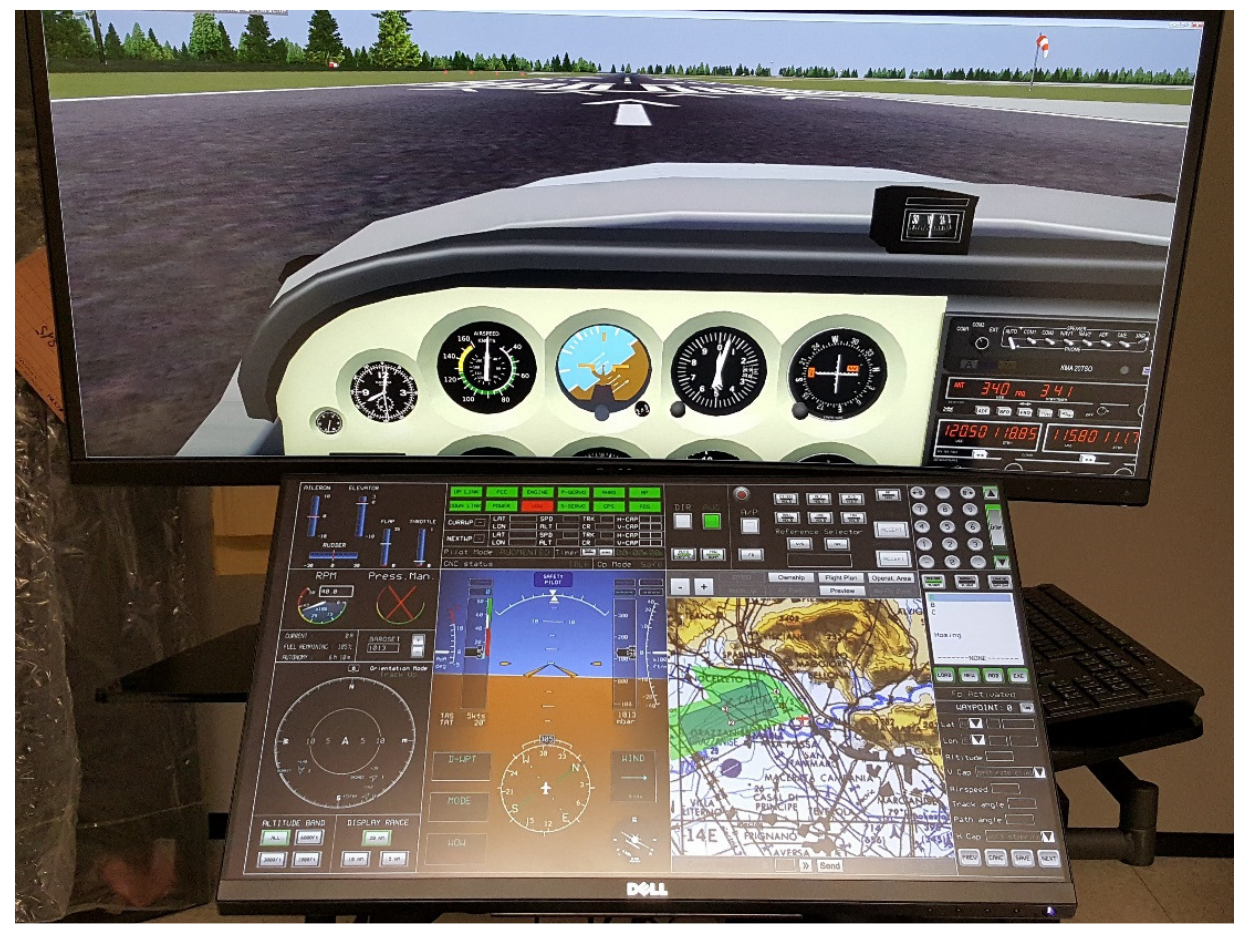

Fig. 7 - GRPS Virtual Cockpit and OTW Displays.

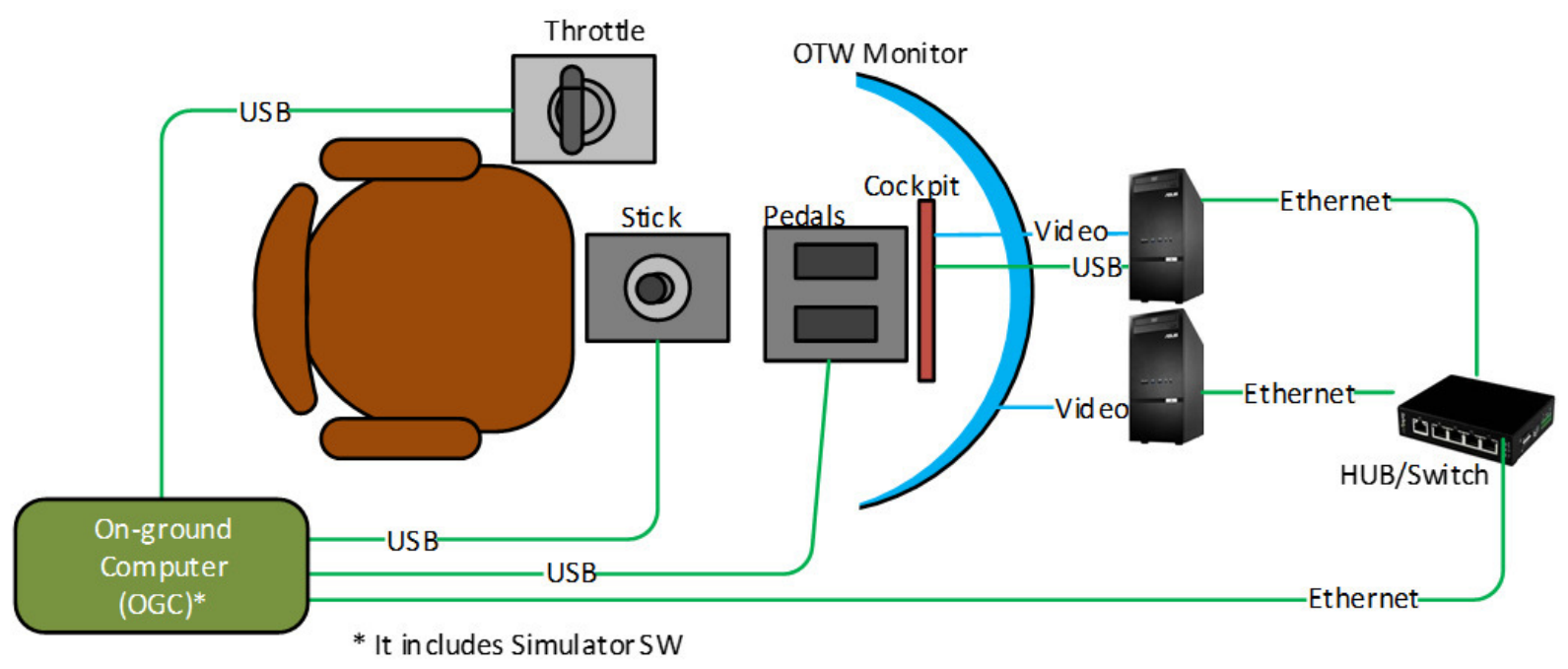

Fig. 8 - GRPS Layout.

The test results are shown in Fig. 9, Fig. 10, and Fig. 11. In Fig. 9 the track angle test maneuver is highlighted in green, meanwhile the pitch angle maneuver is highlighted in red. Fig. 10 shows the time histories of track angle, TAS (True Air Speed), and roll angle, during the track angle test maneuver. Fig. 11 shows the time histories of pitch angle, TAS, and vertical speed, during the pitch angle test maneuver. The track angle is referenced in runway-axes rather than in north-east-down axes. 


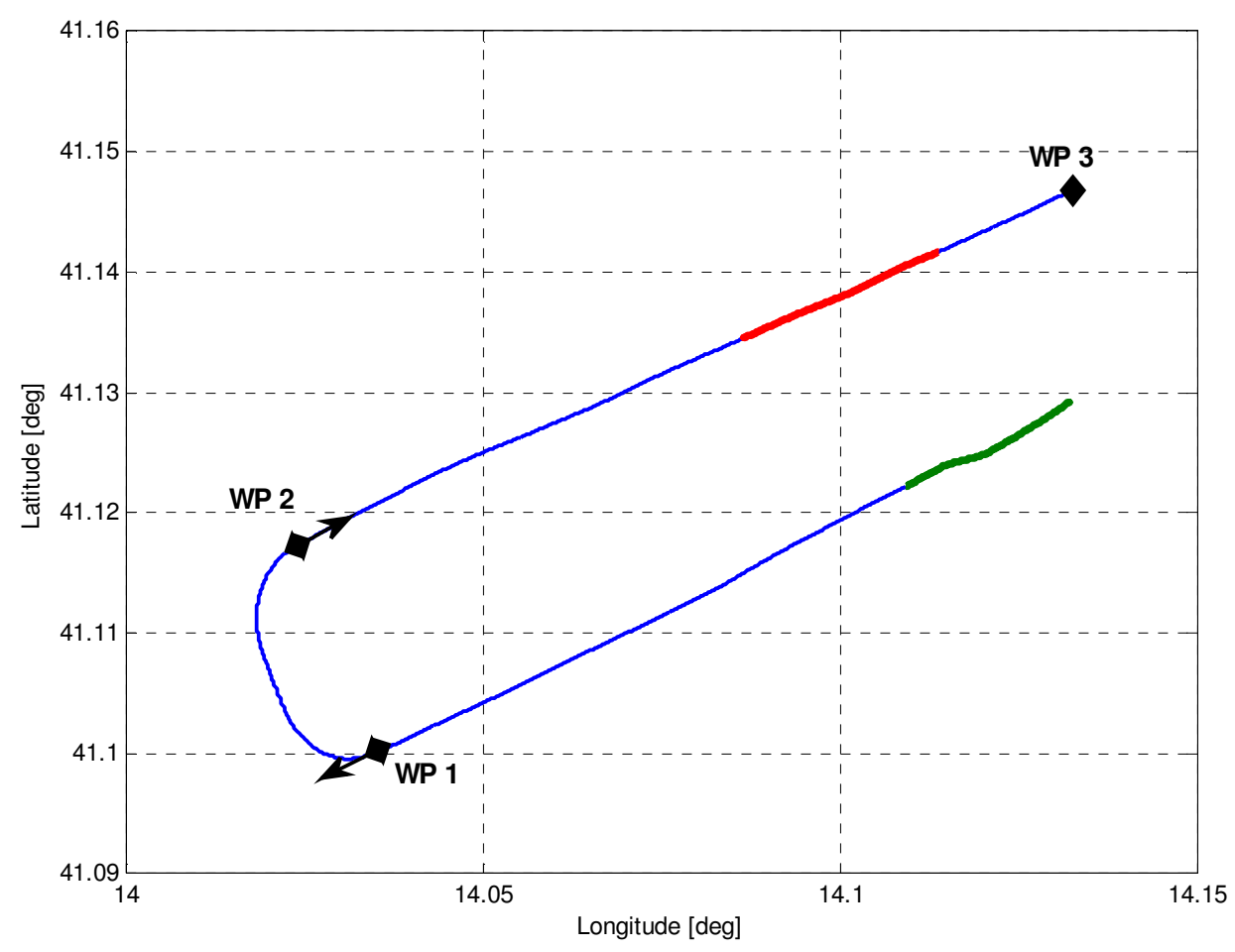

Fig. 9 - RT HIL Test Result: Top View.
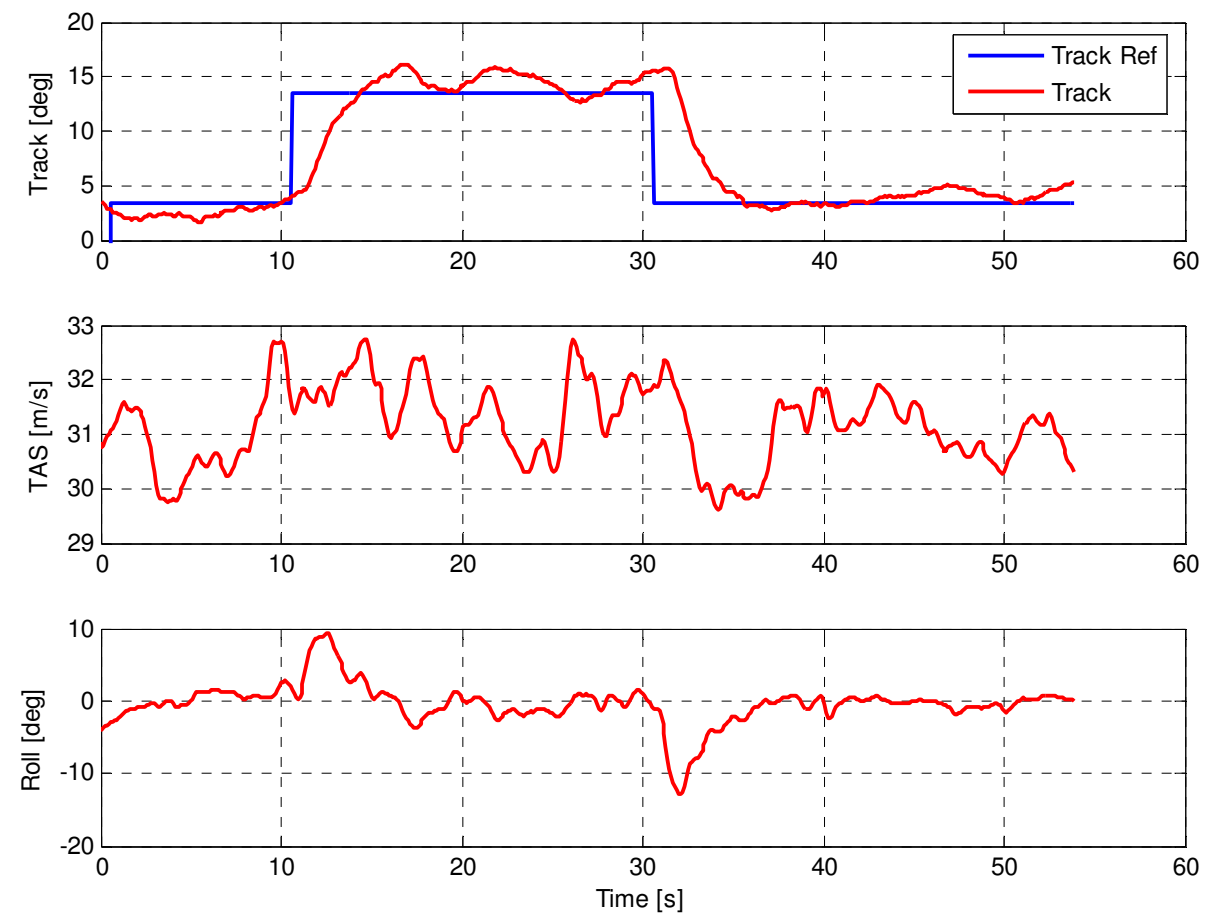

Fig. 10 - RT HIL Test Result: Track Angle Maneuver. 

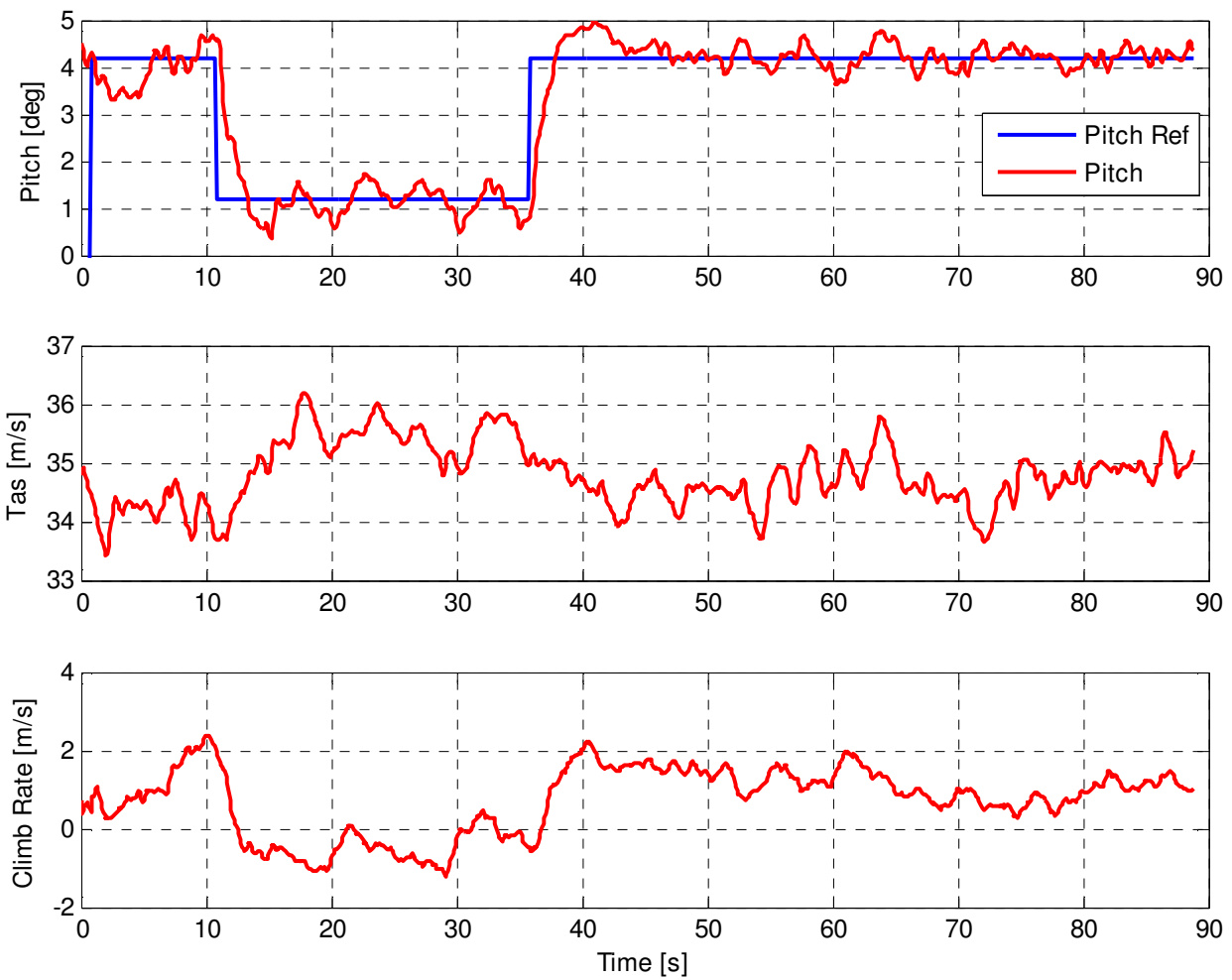

Fig. 11 - RT HIL Test Result: Pitch Angle Maneuver.

\section{Conclusion}

In this paper, a SW framework for an Autopilot with automated test capabilities for supporting the Clean Sky 2 Dynamically Scaled Vehicle flight demonstrations has been presented. Two campaigns of flight tests are expected to be performed over the next two years on two different versions of the flight demonstrator both in Holland and in Italy.

The presented Autopilot, beyond typical basic features already available in commercial products, introduces new characteristics specifically designed for supporting scaled flight testing on unconventional $\mathrm{A} / \mathrm{C}$ (aircraft) configurations, allowing implementation of advanced control laws architectures and strategies.

To facilitate the flight tests, the framework integrates a dedicated SW function for automated flight tests, so avoiding, as much as possible, manual piloting and increasing test condition repeatability. The functional architecture has been developed to be compatible with the highest levels of autonomy. This goal has been pursued by developing a modular and scalar architecture and by adding the capability to execute complex automatic functions based on a simple flight instruction list defined by the remote pilot or the ground operator. To this end, the developed programming meta-language would allow performing mission tasks with better efficiency and effectiveness.

Starting from the architecture described in this paper and exploiting the framework developed, other autonomous functions will be added in the future, based on a preliminary analysis on which level of autonomy those functions are required to achieve. Moreover, some additional advanced control law functionalities will be integrated for managing future versions of the Scaled Vehicle demonstrator.

\section{Acknowledgments}

The authors thank CSJU (Clean Sky Joint Undertaking) for funding the project Clean Sky 2 Large Passenger Aircraft Platform 1 WP1.3 under which the activities described in this paper have been carried out. 


\section{References}

Periodicals

[1] Beer, J. M., et al., "Toward a Framework for Levels of Robot Autonomy in Human-Robot Interaction", Journal of HumanRobot Interaction, Vol. 3, No. 2, Pages 74-99, DOI 10.5898/JHRI.3.2.Beer, 2014

Books

[2] Boyd, J. R., "The Essence of Winning and Losing", 1996

[3] Sheridan, T. B., "Telerobotics, Automation, and Human Supervisory Control”, The MIT Press, 1992

Reports

[4] Proud, R. W., et al., "Methods for Determining the Level of Autonomy to Design into a Human Spaceflight Vehicle: A Function Specific Approach", NASA, 2003

[5] Canadian Army Land Warfare Centre, "No Man's Land: Tech Considerations for Canada's Future Army", 2014

[6] Hew, P. C., "The Generation of Situational Awareness within Autonomous Systems - A Near to Mid Term Study - Issues", Australian Government, Department of Defence, DSTO-GD-0467, 2006

[7] Hew, P. C., "The Generation of Situational Awareness within Autonomous Systems - A Near to Mid Term Study Analysis", Australian Government, Department of Defence, DSTO-GD-1896, 2006

Proceedings

[8] Clough, B. T., "Metrics, Schmetrics! How The Heck Do You Determine A UAV's Autonomy Anyway?", Proceedings of the Performance Metrics for Intelligent Systems Workshop, Gaithersburg, Maryland, 2002.

[9] Huang, H.M., Messina, E., Albus, J., "Toward a Generic Model for Autonomy Levels for Unmanned Systems (ALFUS)", National Institute of Standards and Technology, 2004

[10] Huang, H.M., Pavek, K., Albus J., Messina, E., "Autonomy Levels for Unmanned Systems (ALFUS) Framework: An Update", 2005 SPIE Defense and Security Symposium, 2005

Commercial and Open Products

[11] Micropilot Products - http://www.micropilot.com/products.htm

[12] ArduPilot Products - http://www.ardupilot.co.uk/

[13] Open Pilot Products - http://www.openpilot.org/ 


\section{- Acknowledgement}

This project has received funding from the Clean Sky 2 Joint Undertaking (JU) under grant agreement No 807097. The JU receives support from the European Union's Horizon 2020 research and innovation programme and the Clean Sky $2 \mathrm{JU}$ members other than the Union.

\section{- Disclaimer}

The results, opinions, conclusions, etc. presented in this work are those of the author(s) only and do not necessarily represent the position of the JU; the JU is not responsible for any use made of the information contained herein.
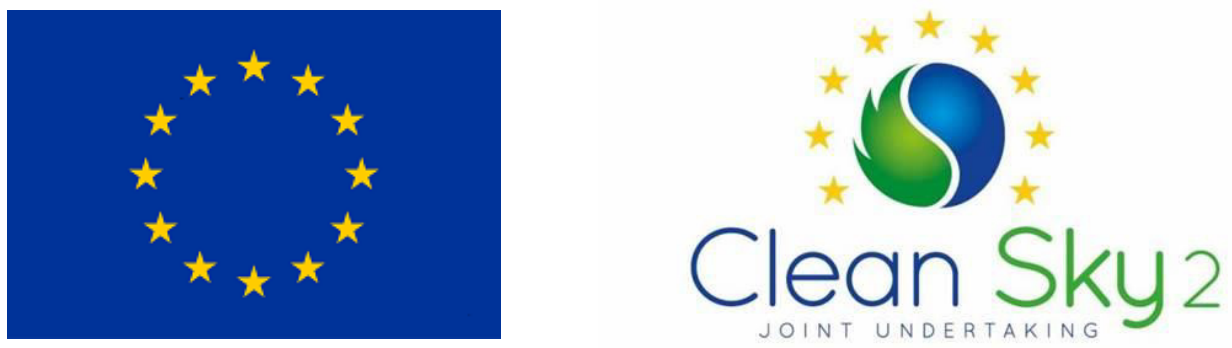